\title{
XXXVI. Relativity and electrodynamics
}

\section{G.W. Walker M.A. F.R.S. A.R.C.Sc.}

To cite this article: G.W. Walker M.A. F.R.S. A.R.C.Sc. (1918) XXXVI. Relativity and electrodynamics, Philosophical Magazine Series 6, 35:208, 327-338, DOI: 10.1080/14786440408635768

To link to this article: http://dx.doi.org/10.1080/14786440408635768

$$
\text { 曲 Published online: } 08 \text { Apr } 2009 .
$$

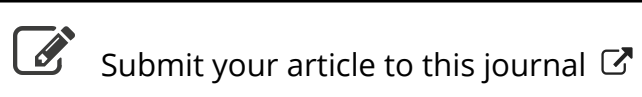

Џ Article views: 10

Q View related articles 두 


\section{$\left[\begin{array}{ll}327 & ]\end{array}\right.$}

XXXVI. Relativity and Electrodynamics. By G. W. WALKER, M.A., F.R.S., A.R.C.Sc., formerly Fellow of Trinity College, Cambridge".

[Plate X.]

GIR OLIVER LODGE'S recent papers in the Philoso$\$$ phical Magazine have brought into prominence once more the difference of attitude of the protagonists in "Relativity Doctrine" and "Newtonian Dynamics." That Sir Oliver's equation of motion for a moving planet requires some amplification in order to take full account of the special features of electrical inertia, will be recognized, and Prof. Eddington has suggested a method of dealing with the problem. Unfortunately, Eddington's method introduces an assumption which is frequently mado by relativists in dealing with electrical inertia, and which in my opinion is inconsistent with the fundamental equations of electrodynamics. In former papers I have drawn attention to this assumption, which is closely linked with the "quasi-stationary principle," and I had not intended to raise the point again. But Sir Oliver has suggested to me that an exposition of my views as to the parting of the ways between the logical development of electrodynamics and the doctrine of relativity would be of value, and I have agreed to his request. My remarks must, however, be confined to electric inertia, and I do not propose to enter on the gravitational and astronomical developments of Einstein's hypothesis.

'l'he main point at issue may, I think, be put very concisely. Relativists assume that "the kinetic energy of a moving electrical system is a function of the resultant speed only and is independent of the direction of motion."

My thesis is that this assumption is not consistent with the fundamentil electromagnetic equations for the xether (supposed immobile), and that " the energy, or preferably the modified Lagrangean function, depends on the acceleration as well as on the speed of the system and involves also the relative direction of these."

While the above appears to me to be the main point, there is no doubt that subsidiary considerations arise. Theory and experiment have interacted in a curious way, and I think the discussion should proceed by taking notice of the historical development. Sir Joseph Thomson was the first to

* Communicated by Sir Oliver Lodge. 
prove theoretically that a moving electrified system would possess inertia, which Heaviside showed would depend on the speed with which the system moves. A later calculation by Thomson referred to a particular form of nucleus and to the momentum which it would carry with it in virtue of a uniform translation. It is extremely important to realize that the character of the nucleus determines the manner in which the speed enters in the expression for the momentum or for the energy. It is also vital to realize that while the momentum or the energy can be calculated for a particular form of nucleus moving with a uniform speed, it has not so far been found possible to give a complete solution when the speed is variable.

M. Abraham extended Thomson's calculations, and he assumed that while the nucleus was still a sphere it was a perfect conductor, and he consequently obtained a value for the momentum in a state of uniform translation which differed from that found by Thomson when squares of the speed were retained. He emphasized the distinction between the effective inertia for acceleration along and perpendicular to the direction of motion.

But finding that he could not obtain the exact solution for a variable speed, Abraham made use of what is called the "quasi-stationary principle," which amounts to saying that if we can calculate the momentum, or if we prefer it the Lagrangean function, for a uniform motion we can infer the equations of motion for a small departure from this state in the ordinary way. My contention is that we can no more do this logically for electromagnetic systems than wo can for ordinary dynamical systems. We know quite well that we do not get the correct equations for small departures from a steady state, when the steady motion values are inserted in the Lagrangean function before the differential equations of motion are formed. The steady motion values may be inserted after the equations have been formed from the general Lagrangean function.

Abraham calculated expressions for longitudinal and transversal electric inertia by means of the quasi-stationary principle. Experiments on transverse inertia became possible with the discovery of the Becquerel rays, and of the minute negatively charged particles projected from radium with speeds only little short of that of light.

The matter was taken up first by W. Kaufmann, and I have a special personal interest in this since I was working side by side with him in the laboratory at Göttingen while his experiments were in progress. 
Kanfmann deflected the particles by crossed electric and magnetic fields, by which the particles are sifted out according to their speed, so that the ends of their trajectories form a curve on a photographic plate.

Kaufmann considered that his measurements proved that Abraham's expression for transverse inertia was correct, and that the inertia of the particles was purely electromagnetic in origin.

We must now retrace our steps to consider the important contributions to the theory of moving systems made by Prof. H. A. Lorentz and Sir Joseph Larmor. They proved that a mathematical correlation held between an electrical system at rest and a certain system maintained in uniform translation. "If the moving system has a uniform speed $k c$ (where $c$ is the velocity of light) in the direction $x$, and the $x$ linear extent of the moving system is $\left(1-k^{2}\right)^{\frac{1}{2}}$ of the linear extent of the fixed system, and the variables time $t$ and distance $x$ in the fixed system are transformed to $t^{\prime}$ and $x^{\prime}$ in the moving system by a certain linear transformation involving $k$, then the state of the fixed system in terms of $t$ and $x$ is the same as that of the moving system in terms of $t^{\prime}$ and $x^{\prime}$."

It is reasonable to inquire if the contraction in the proportion $\left(1-k^{2}\right)^{\frac{1}{2}}$ actually takes place when a system at rest is put into uniform translation, for if so it provides an explanation of the Michelson-Morley experiment.

Now it is quite certain that the mathematical transformation is not true when $k$ is variable, and therefore not true at any intermediate stage by which the system at rest might conceivably pass to the correlated system in uniform motion, if it evor does so at all. But relativists have assumed that the correlation proved by Lorentz and Larmor for a uniform translation only is true, and that the change actually takes place, when the speed is variable. It appears to me that if the primary equations are correct, the assumption is not merely not permissible, but is not true; and, on the contrary, if the assumption does represent actual truth, then the primary equations are wrong and must go. We await proof, which so far has not been offered.

The longitudinal and transverse inertia of a "contracted" electron have been calculated by the quasi-stationary method, so that there is a double source of error in the result.

Experiments by Kaufmann, Bestelmeyer, and others have been offered as experimental proof that the formula for transverse inertia of a contracted electron on relativity doctrine is correct. My contention is that while the experiments do not conflict with the relativity formula, the 
formula is inconsistent with the electrodynamic equations, and that several other formula correctly deduced from the primary equations agree with the experiments equally well.

I doubt if many people in this country realize the very meagre character of the experimental results, and $I$ therefore give a full-sized reproduction (Pl. X.) of the photographic plate from which Kaufmann made his measurements. The electric defiexion is across the paper and the magnetic. deflexion up the paper, and it may be pointed out that if the inertia of the particles were quite independent of speed, the small curved arcs would be parabolas, and that it is only in so far as these arcs differ from parabolas that any dependence of inertia on speed can be made out at all. Further, the highest speed particles are those for which the deflexion is least.

I now return to the theoretical treatment of electric inertia. In order to avoid the error of the quasi-stationary principle, I developed some time ago a method of obtaining the longitudinal and transversal inertia directly from the primary equations by Newtonian methods. The method is rather tedious, but its correctness has not been called in question. Its application is general, but to get definite results the character of the nucleus must be specified. Various systems may be examined provided they do not violate any fundamental restriction imposed by electrodynamic conditions. In this way I examined the nucleus assumed by Sir. Joseph Thomson and was able to confirm his result for transverse inertia, but obtained a different result for longitudinal inertia. On the other hand, with the nucleus assumed by Abraham I was able to confirm his result for longitudinal inertia, but not that for transverse inertia.

Again, recently I examined the case of a contracted conducting spheroid which agreed in form with Lorentz's contracted electron for the uniform speed, but did not alter its form when acceleration was imposed *. The results for both longitudinal and transverse inertia differ from those adopted by relativists.

The differences that arise in these examples only become important when squares and higher terms in the speed are retained, and they arise from the fact that when acceleration is imposed, additional electric forces are set up which have to be allowed for in utilizing the boundary conditions at

* The restriction is unnecessary, as I now find that my results are not altered when the surface deforms under acceleration as Lorentz assumes. 
the surface of the electron. Thus, for instance, with a conductor there is a redistribution of the charge, which depends on the acceleration, the speed, and the direction of the acceleration relative to that of the speed. So the Lagrangean function must involve these things, and I am doubtful if it is the resultant speed that alone enters.

The results I have obtained for electric inertia by my direct, if pedestrian, method, can be shown to prove that the energy cannot be expressed as a function of resultant speed only. For if

$$
\mathrm{T}=f\left(v^{2}\right)
$$

we find that in the direction of motion, say along $x$, we get

$$
\frac{d}{d t} \frac{d \mathrm{~T}}{d \dot{x}}=\ddot{x}\left(2 f^{\prime}+4 v^{2} f^{\prime \prime}\right),
$$

and at right angles to this, say along $y$, we get

$$
\frac{d}{d t} \frac{d \mathbf{T}}{d \dot{y}}=\ddot{y} 2 f,
$$

therefore longitudinal inertia $=m_{1}=2 f^{\prime}+4 \boldsymbol{v}^{2} f^{\prime \prime}$,

and transversal inertia $=m_{2}=2 f^{\prime}$.

Hence. since

$$
\begin{gathered}
\frac{d}{d v} m_{2} v=\frac{d}{d v} 2 v f^{\prime}=2 f^{\prime}+4 v^{2} f^{\prime \prime}, \\
m_{1}=\frac{d m_{2} v}{d v}
\end{gathered}
$$

must be satisfied. But it does not follow that $\mathrm{T}=f\left(v^{2}\right)$ if $m_{1}=\frac{d m_{2} v}{d v}$ is satisfied.

My results for the cases mentioned do not satisfy this condition, and unless it can be shown directly from the primary equations that arithmetical error has entered into my calculations, it follows that the kinetic energy of a system in variable motion is not expressible as a function of the resultant speed only.

The conclusion is that Eddington's proposed treatment of the astronomical problem is invalid, and I see no help for it but to start with the equations in the tangential form,

$$
\begin{gathered}
m_{1} v d v=\mathrm{T}, \\
m_{2} v^{2} / \rho=\mathrm{N},
\end{gathered}
$$

where $m_{1}$ and $m_{2}$ are different functions of $v^{2}$, which can be calculated when the electrical system is fully specified. 
Starting thus with longitudinal and transverse electric inertia given by $m_{1}$ and $m_{2}$ as functions of the resultant speed, and employing what I hold to be the correct procedure in forming the equations of motion, viz., resolving along and perpendicular to the resultant direction of rotion, we can proceed as follows:-

Let the origin be the sun moving in space with components of velocity $u, v, w$, which are constant, and let $x, y, z$ be the co-ordinates of a planet relative to the sun and referred to axes through the sun. The components of relative velocity of the planet are

$$
\dot{x}, \quad \dot{y}, \quad \dot{z},
$$

and of velocity in space $\dot{x}+u, \dot{y}+v, \dot{z}+w$, with resultant say $V$.

The components of acceleration are

$$
\ddot{x}, \ddot{y}, \ddot{z} .
$$

The acceleration along the resultant direction of velocity is

$$
\{(\dot{x}+u) \ddot{x}+(\dot{y}+v) \ddot{y}+(\dot{z} \pm w) \ddot{z}\} / V,
$$

and if the components of foree are $X, Y, Z$, the component along the direction of $\mathrm{V}$ is

$$
\{\mathrm{X}(\dot{x}+u)+\mathrm{Y}(\dot{y}+v)+\mathrm{Z}(\dot{z}+w)\} / \mathrm{V} .
$$

Hence the equation

$$
\begin{aligned}
m_{1}\{(\dot{x}+u) \ddot{x}+(\dot{y}+v) \ddot{y}+ & (\dot{z}+w) \ddot{z}\} \\
& =\mathrm{X}(\dot{x}+u)+\mathrm{Y}(\dot{y}+v)+\mathrm{Z}(\dot{z}+w) \\
& =\mathrm{S} \text { say. . . . . . (1) }
\end{aligned}
$$

Resolve along any direction $\lambda, \mu, v$, at right angles to the direction of $V$,

then

$$
m_{2}\{\lambda \ddot{x}+\mu \ddot{y}+\nu \ddot{z}\}=\mathrm{X} \lambda+\mathrm{Y} \mu+\mathrm{Z} \nu,
$$

and

$$
(\dot{x}+u) \lambda+(\dot{y}+v) \mu+(\dot{z}+w) \nu=0 \text {. }
$$

Hence

$$
\begin{aligned}
& m_{2} \ddot{x}=\mathrm{X}+(\dot{x}+u) k, \\
& m_{2} \ddot{y}=\mathrm{Y}+(\dot{y}+v) k, \\
& m_{2} \ddot{z}=\mathrm{Z}+(\dot{z}+w) k,
\end{aligned}
$$

where $k$ is some quantity to be determined. Multiply these 
in order by $\frac{m_{1}}{m_{2}}(\dot{x}+u)$, otc., and add and use (1). Then we find

$$
\mathrm{S}=\frac{m_{1}}{m_{2}} \mathrm{~S}+\frac{m_{1}}{m_{2}} k \mathrm{~V}^{2}
$$

or

$$
k=\frac{\left(m_{2}-m_{1}\right)}{m_{1}} \mathrm{~S} / \mathrm{V}^{2} \text { where } \mathrm{V}^{2}=(\dot{x}+u)^{2}+(\dot{y}+v)^{2}+(\dot{z}+w)^{2} .
$$

Hence the Cartesian equations of motion are

$$
\begin{aligned}
& m_{2} \ddot{x}=\mathrm{X}+\frac{\left(m_{2}-m_{1}\right)}{m_{1}} \frac{(\dot{x}+u)}{\mathrm{V}^{2}}\{\mathrm{X}(\dot{x}+u)+\mathrm{Y}(\dot{y}+v)+\mathrm{Z}(\dot{z}+w)\}, \\
& m_{2} \ddot{y}=\mathrm{Y}+\frac{\left(m_{2}-m_{1}\right)}{m_{1}} \frac{(\dot{y}+v)}{\mathrm{V}^{2}}\{\mathrm{X}(\dot{x}+u)+\mathrm{Y}(\dot{y}+v)+\mathrm{Z}(\dot{z}+w)\}, \\
& m_{2} \ddot{z}=\mathrm{Z}+\frac{\left(m_{2}-m_{1}\right)}{m_{1}} \frac{(\dot{z}+w)}{\mathrm{V}^{2}}\{\mathrm{X}(\dot{x}+u)+\mathrm{Y}(\dot{y}+v)+\mathrm{Z}(\dot{z}+w)\} .
\end{aligned}
$$

Also for a central attraction

$$
\mathrm{X}=-\mu x / r^{3}, \quad \mathrm{Y}=-\mu y / r^{3}, \quad \mathrm{Z}=-\mu z / r^{3} .
$$

From these it appears that a planet's orbit cannot remain a plane orbit during transference through the other, except in the special case when the direction of the sun's motion is in and remains in the plane of the orbit.

We can readily transform the equations to polars since the disturbance is along the direction of the resultant velocity in space.

For the example under recent discussion by Sir Oliver Lodge and as a first approximation, we might take

$$
m_{1}=m_{0}\left(1+a \mathrm{~V}^{2} / c^{2}\right), \quad m_{2}=m_{0}\left(1+b \mathrm{~V}^{2} / c^{2}\right),
$$

and neglecting squares of $\mathrm{V}^{2} / c^{2}$, treat the problem as one of a disturbed central orbit.

Astronomers doubtless know the best mode of dealing with this, and could obtain a solution withont difficulty.

It is important to remember that these equations are first approximations only to the general equations connecting moving material systems with the state of the ather.

A special case amenable to elementary treatment is that 
of the orbit of a particle, having electric inertia, round a fixed centre which is at rest in space.

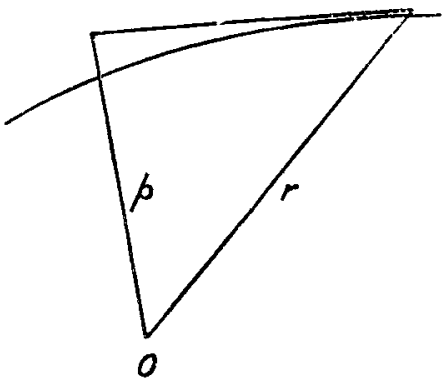

Let $v=$ resultant velocity at any point of the orbit.

$\rho=$ radius of curvature.

$r=$ radius vector.

$p=$ perpendicular on tangent.

$\mu / r^{2}=$ attraction to the centre.

$m_{1}=$ longitudinal inertia $=m_{0}\left(1+k_{1} v^{2} / c^{2}\right)$.

$m_{2}=$ transverse inertia $=m_{0}\left(1+k_{2} v^{2} / c^{2}\right)$ neglecting squares of $v^{2} / c^{2}$.

The orbit is plane, and resolving along and perpendicular to the path we get

and

$$
m_{1} v \frac{d v}{d s}=-\frac{\mu}{r^{2}} \cdot \frac{d r}{d s}, \quad . \quad . \quad . \quad .
$$

Integrating (1) we get

$$
m_{0}\left(v^{2}+\frac{1}{2} k_{1} v^{4} / c^{2}\right)=\frac{2 \mu}{r}-\frac{\mu}{a},
$$

where $a$ is a constant.

From (2)

$$
m_{0}\left(v^{2}+k_{2} v^{4} / c^{2}\right)=\frac{\mu p}{r^{2}} \frac{d r}{d p}, \quad \text { since } \rho=r \frac{d r}{d p} .
$$

Let $u=1 / r$ and $u_{0}=1 / a$,

$$
\therefore m_{0}\left(v^{2}+\frac{1}{2} k_{1} v^{4} / c^{2}\right)=2 \mu u-\mu u_{0},
$$

and

$$
m_{0}\left(v^{2}+k_{2} v^{4} / c^{2}\right)=-\mu p \frac{d u}{d p},
$$


$\therefore$ approximately $m_{0} v^{2}=2 \mu u-\mu u_{0}-\frac{1}{2} \frac{k_{1}}{m_{0} c^{2}}\left(2 \mu u-\mu u_{0}\right)^{2}$,

and

$$
\left(2 \mu u-\mu u_{0}\right)+\frac{\left(k_{2}-\frac{1}{2} k_{1}\right)}{m_{0} c^{2}}\left(2 \mu u-\mu u_{0}\right)^{2}=-\mu p \frac{d u}{d p},
$$

or putting

$$
x=2 \mu u-\mu u_{0},
$$

and

$$
\lambda=\left(k_{2}-\frac{1}{2} k_{1}\right) / m_{0} c^{2} \text {, }
$$

we get

$$
x+\lambda x^{2}=-\frac{1}{2} p \frac{d x}{d p}
$$

or

$$
-2 \frac{d p}{p}=\delta x\left\{\frac{1}{x}-\frac{\lambda}{1+\lambda x}\right\},
$$

$\therefore$ integrating we get

$$
b^{2} / p^{2}=\frac{x}{1+\lambda x}=x-\lambda x^{2} \text { neglecting squares of } \lambda,
$$

where $b$ is a constant of integration.

Now

$$
\begin{gathered}
\frac{1}{p^{2}}=u^{2}+\left(\frac{d u}{d \theta}\right)^{2}, \\
\therefore \quad b^{2}\left\{u^{2}+\left(\frac{d u}{d \theta}\right)^{2}\right\}=\left(2 \mu u-\mu u_{0}\right)-\lambda\left(2 \mu u-\mu u_{0}\right)^{2}, \\
b^{2}\left(\frac{d u}{d \theta}\right)^{2}=\left(2 \mu u-\mu u_{0}\right)-\lambda\left(2 \mu u-\mu u_{0}\right)^{2}-b^{2} u^{2},
\end{gathered}
$$

so that the integral is of the form

$$
l u=1+e \cos \left[\left\{1+\frac{4 \mu^{2} \lambda}{b^{2}}\right\}^{\frac{1}{3}} \theta-\eta\right],
$$

where $\eta$ is an arbitrary constant and $l$ and $e$ are determinate constants in $\mu, \alpha, b$, and $\lambda$. This solution implies an elliptic orbit slowly revolving in its own plane. The eccentrieity does not change, but the apses will advance in the direction of description of the orbit by

$$
\frac{2 \pi}{\left\{1+\frac{4 \mu^{2} \lambda}{b^{2}}\right\}^{\frac{1}{2}}}-2 \pi \text { for each description of the orbit, }
$$

i. e. since $\lambda$ is small,

by

$$
\begin{aligned}
& -\frac{4 \pi \mu^{2} \lambda}{b^{2}}, \\
& \frac{4 \pi \mu^{2}}{m_{0} b^{2}}\left(\frac{1}{2} k_{1}-k_{2}\right) / c^{2} .
\end{aligned}
$$

or 
Expressed in terms of the semi-major axis $R_{0}$, eccentricity $e$ and periodic time $T$, the progress per revolution is

$$
\left\{\frac{2 \pi \mathrm{R}_{0}}{\mathrm{~T}\left(1-e^{2}\right)^{\frac{1}{2}}}\right\}^{2} \frac{\left(\frac{1}{2} k_{1}-k_{2}\right)}{c^{2}} \cdot 4 \pi .
$$

The apses therefore progress or regress according as $\left(\frac{1}{2} k_{1}-k_{2}\right)$ is positive or negative.

We have no knowledge as to the proper forms of $m_{1}$ and $m_{2}$ for matter in bulk, but the following are results for hypothetical single nuclei.

For the contracted electron using relativity methods,

$$
m_{1}=m_{0}\left(1+\frac{3}{2} v^{2} / c^{2}\right), \quad m_{2}=m_{0}\left(1+\frac{1}{2} v^{2} / c^{2}\right),
$$

so that $\frac{1}{2} k_{1}-k_{2}=\frac{1}{4}$ (or progression).

From the primary electromagnetic equations my results* are :-

For the contracted conducting electron :

$$
m_{1}=m_{0}\left(1+\frac{13}{10} v^{2} / c^{2}\right), \quad m_{2}=m_{0}\left(1+\frac{41}{60} v^{2} / c^{2}\right),
$$

so that

$$
\frac{1}{2} k_{1}-k_{2}=-\frac{1}{30} \text { (or regression). }
$$

For Thomson's electron with special surface condition :

$$
m_{1}=m_{0}\left(1+\frac{7}{10} v^{2} / c^{2}\right), \quad m_{2}=m_{0}\left(1+\frac{4}{10} v^{2} / c^{2}\right),
$$

so that

$$
\frac{1}{2} k_{1}-k_{2}=-\frac{1}{20} \text { (or regression). }
$$

For spherical conductor which does not change in shape :

$$
m_{1}=m_{0}\left(1+\frac{6}{5} v^{2} / c^{2}\right), \quad m_{1}=m_{0}\left(1+\frac{19}{60} v^{2} / c^{2}\right),
$$

so that

$$
\frac{1}{2} k_{1}-k_{2}=\frac{17}{60} \text { (or progression). }
$$

This last case is numerically almost the same as that for the contracted electron by relativity methods. This is important, because it shows that so far as inertia enters in the astronomical problem we can get practically the same result

* Proved only for disturbance from a steady state. 
as a logical sequence from the fundamental equations as has been obtained by relativity doctrine.

Our results for the apsidal progression per revolution are: Contracted electron by relativity method,

$$
\left\{\frac{2 \pi \mathrm{R}_{0}}{\mathrm{~T}\left(1-e^{2}\right)^{\frac{1}{2}}}\right\}^{2} \frac{\pi}{c^{2}} \text {. }
$$

Spherical electron by orthodox method,

$$
\left\{\frac{2 \pi \mathrm{R}_{0}}{\mathrm{~T}\left(1-e^{2}\right)^{\frac{3}{2}}}\right\}^{2} \frac{17}{15} \frac{\pi}{e^{2}}
$$

Now Einstein obtained

$$
\left\{\frac{2 \pi \mathrm{R}_{0}}{\mathrm{~T}\left(1-e^{2}\right)^{\frac{3}{2}}}\right\}^{2} \frac{6 \pi}{c^{2}}
$$

which numerically is in close agreement with observations on "Mercury."

This result is obtained by assuming that the attraction depends on the velocity. It is easily seen from our analysis that if

$$
\mu=\mu_{0}\left(1+k_{3} v^{2} / c^{2}\right),
$$

we get for the apsidal progress

$$
\left\{\frac{2 \pi \mathbf{R}_{0}}{\mathrm{~T}\left(1-e^{2}\right)^{\frac{1}{2}}}\right\}^{2} \frac{4 \pi}{c^{2}}\left(\frac{1}{2} k_{1}+\frac{k_{3}}{2}-k_{2}\right) .
$$

In order to get the observed value for "Mercury" $k_{3}$ would have to be $5 / 2$ if $\frac{1}{2} k_{1}-k_{2}$ is $1 / 4$.

It is important to recognize that it is only by introducing either explicitly or implicitly this comparatively large dependence on speed, of the attraction between bodies that Einstein can get the numerical agreement. Such dependence based on the known forces between electrical currents has been recognized before now in the theory of electrodynamics, but is hardly acceptable in gravitational theory. On these lines it appears that ortbodox electrodynamics is quite as capable of providing an explanation of this astronomical feature as Einstein's theory. It is, however, important that endeavour should be made to determine, if possible, the numerical value of $\frac{1}{2} k_{1}+\frac{1}{2} k_{3}-k_{2}$ for matter in bulk.

There still remains the question of the effect of transference in space as suggested by Sir Oliver Lodge. Eddington's conclusions on this problem may be modified considerably 
when what I hold to be more correct equations of motion are used.

NotE.-At Sir Oliver Lodge's request I have calculated $m_{1}$ and $m_{2}$ for Bucherer's electron which has the same form as Lorentz's electron but keeps its volume unchanged. My results are

$$
m_{1}=m_{0}\left(1+\frac{17}{15} v^{2} / c^{2}\right), \quad m_{2}=m_{0}\left(1+\frac{31}{6 \overline{0}} v^{2} / c^{2}\right),
$$

so that

$$
\frac{1}{2} k_{1}-k_{2}=\frac{1}{20} \text {. }
$$

XXXVII. Molecular Frequency and Molecular Number. By H. Stanley Ali.en, M.A., D.Sc., University of London, King's College*.

Part I.

\section{\$1. Molecular Number.}

T THE work of Moseley on the high-frequency spectra of 1 the elements has established securely the importance of the "atomic number" of an element: that is, the number which determines the place in the periodic classification and fixes the charge carried by the central part of the atom. It is now certain that the atomic number is more fundamental than the atomic weight. Recent investigations of the atomic weight of lead of radioactive origin have shown that the value obtained for this quantity depends upon the source from which the material is derived. An interesting account of these researches has been given by Soddyt, who points out that the atomic weight as ordinarily understood is not the unique quantity hitherto supposed. In the future increasing importance will be attached to the atomic number. It is the conviction of the present author that this will prove true not only in connexion with the properties of the chemical elements but also in dealing with compounds. In the latter case it is convenient to introduce the term "molecular number" to signify the sum of the positive charges carried by the atomic nuclei contained in the molecule. Thus when a molecule contains $a$ atoms of an element $\mathbf{A}, b$ atoms of $\mathbf{B}$, $c$ atoms of $\mathrm{C}$, so that its chemical formula is $\mathrm{A}_{a} \mathrm{~B}_{b} \mathrm{C}_{c}$, the molecular number $\mathrm{N}=a \mathrm{~N}_{a}+b \mathrm{~N}_{b}+c \mathrm{~N}_{c}$, where $\mathrm{N}_{a}, \mathrm{~N}_{b}, \mathrm{~N}_{c}$ are the atomic numbers of the component elements. For

* Communicated by the Author.

† Royal Institution Lecture, 'Nature,' vol. xcix. p. 414 (1917). 
WATKER.

Phil. Mag. Ser. 6, Vol. 35. Pl. X.

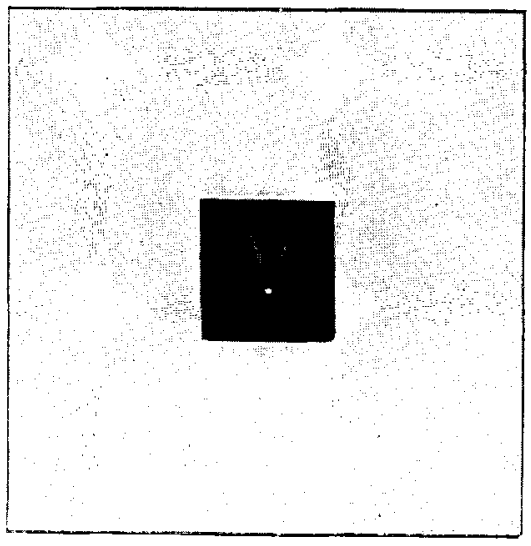

